\title{
A Wavelet-Based Method for Non-Invasive Dominant Frequency Detection in Atrial Fibrillation
}

\author{
Victor G Marques ${ }^{1}$, Miguel Rodrigo ${ }^{2}$, María S Guillem ${ }^{2}$, João Salinet ${ }^{1}$ \\ ${ }^{1}$ Federal University of ABC, São Bernardo do Campo, Brazil \\ 2 ITACA Institute, Universitat Politècnica de València, Spain
}

\begin{abstract}
Atrial dominant frequency $(D F)$ maps undergoing atrial fibrillation $(A F)$ presented good spatial correlation with those obtained with the non-invasive body surface potential mapping (BSPM). In this study, a robust BSPM-DF calculation method based on wavelet analysis is proposed. Continuous wavelet transform (Gaussian mother wavelet) along 40 scales in the pseudo-frequency range of $3-30 \mathrm{~Hz}$ is performed in each BSPM signal. DFs are estimated from the intervals between the peaks, representing the activation times, in the maximum energy scale. The results are compared with the traditionally widely applied Welch periodogram. The accuracy of both methods was assessed using the absolute errors between BSPM and atrial highest $D F$ (HDFs) and assumed correct if $\leq 1$. The robustness of the methods was tested on different protocols: increasing levels of WGN, artificial DF harmonics presence, and reduction of the number of leads. 11 AF simulations and 12 $A F$ patients are considered in the analysis. The proposed method outperformed the Welch approach, obtaining more correct estimations of atrial HDFs non-invasively in models $(81.82 \%$ vs $45.45 \%)$ and patients $(75.00 \%$ vs $66.67 \%)$ and being more robust to noise and reduction in spatial resolution, thus helping to increase the non-invasive diagnostic ability of BSPM in AF.
\end{abstract}

\section{Introduction}

Atrial fibrillation (AF) is the most common cardiac arrhythmia in clinical practice, being associated with high mortality and morbidity, mainly due to an increased risk of thromboembolic events [1]. The mechanisms behind AF are complex and not completely understood [1]. Recent studies have related the maintenance of AF to localized regions which can then be targeted for ablation, improving the therapy outcomes with personalized approaches $[2,3]$

One of the important biomarkers of AF driving regions is their fast activation rate [4]. Due to the complexity of AF signals, locating driving regions with activation- based time analysis can be challenging, motivating studies in the frequency domain [5]. Investigators related the AF cycle length with the highest power peak in Welch periodograms, defined as the dominant frequency (DF) [4]. The region with the highest DF (HDF) is related to AF drivers and could be targeted in ablation $[4,5]$. HDF areas can be identified non-invasively through the body surface potential mapping (BSPM) DF mapping [6], even with the low-pass filter effect intrinsically related to the volume conductor effect of the torso [7], providing a valuable clinical tool which can be used prior invasive mapping.

Nonetheless, Fourier based DF analysis presents important limitations, such as the lack of the identification of activation times and sensitivity to signal uncertainties (e.g. presence of harmonic activity and dynamic changes in frequency and phase) [5]. A possible alternative for traditional DF analysis is the wavelet transform (WT), which can be understood as a generalization of the Fourier transform where the sine waves are replaced by stretched, squeezed, and translated versions of a single waveform (mother wavelet) [8]. The signal is decomposed in scales rather than frequencies, which are related to a bandwidth, depending on the chosen wavelet. WT is a technique with good localization in both time and frequency domains and has the ability to identify points with close to singular behavior [8], such as the sharp transitions associated with activations in BSPM.

In this study, we proposed a WT-based method for estimating atrial HDFs based on the identification of individual activation times, comparing it with traditional peak detection in Welch periodograms, and testing its robustness is under noisy conditions, presence of harmonics and in different BSPM layouts.

\section{Methods}

A realistic 3D model of the atria was used to generate 11 rotor driven AF simulations with 4 seconds each, localized in different portions of the left and right atria [9]. DF values were determined in each of the 2048 unipolar electrograms (EGM) obtained with the models by detecting the 
highest power peak in Welch periodograms (80\% overlap, zero-padded to achieve a spectral resolution of $0.01 \mathrm{~Hz}$ ) [9]. DF values were manually inspected and compared with the activation counts to avoid inconsistencies. The atrial $\mathrm{HDF}\left(\mathrm{HDF}_{\text {Atria }}\right)$ at the location of the rotor was defined as the driving frequency of AF. BSPM signals were obtained by solving the forward problem with the boundary element method, resulting in 771 nodes with signals referenced to the Wilson central terminal and sampled at $f_{S}=500 \mathrm{~Hz}$ [9]. Waist, neck and arm leads were excluded to represent a realistic measurement, resulting in 567 leads. White Gaussian noise (WGN) was added to the BSPM with a signal-to-noise ratio (SNR) of $60 \mathrm{~dB}$ [9].

Additionally, 67 BSPM leads $\left(f_{S}=2048 \mathrm{~Hz}\right)$ were recorded from 12 patients admitted for ablation of drug refractory paroxysmal $(N=9,8$ male) and persistent $\mathrm{AF}$ ( $N=3$, all male)[6]. Simultaneously, intracardiac bipolar EGMs were acquired in 15 locations from both atria $\left(f_{S}=977 \mathrm{~Hz}\right)$ by using the CARTO navigation system with embedded spectral analysis capabilities (CARTO XP, version 7.7; Biosense-Webster, Diamond Bar, CA). EGMs were band-pass filtered $(40-250 \mathrm{~Hz})$, rectified, and then low-pass filtered $(20 \mathrm{~Hz})$. 4-second segments within the longest RR interval after the administration of adenosine were used for the analysis of atrial EGMs and BSPM. In 3 patients, QRS complexes were canceled, as the RR intervals were shorter than 4 seconds. DFs in atrial EGMs were determined in each location by detecting the peak with the highest frequency power in Welch periodograms after manual inspection to avoid inconsistencies [4]. The $\mathrm{HDF}_{\text {Atria }}$ was determined and its location was defined as the driving region of the arrhythmia in the patients.

The 3D coordinates of the BSPM nodes in the models were mapped to a $2 \mathrm{D}$ configuration by projecting their positions into a cylinder wall and unwrapping it with the left side of the torso in the center of the image. Electrode positions in patients were arranged in a two-dimensional configuration based on the lead layout [6], with distances between adjacent columns and rows were set at $5 \mathrm{~cm}$. The signals from both models and patients were interpolated into a 30 X 65 grid using cubic splines [6]. BSPM signals were filtered, when indicated in the methodology, as following: the baseline was estimated by downsampling the signal to $0.025 \cdot f_{S} \mathrm{~Hz}$ and low-pass filtering at $0.5 \mathrm{~Hz}$ (10th order Butterworth). The baseline was then interpolated to its initial $f_{S}$ and subtracted from the original signals [10]. A low-pass filter (10th order Butterworth) was applied at $30 \mathrm{~Hz}$, covering the spectral range of AF [10].

DFs were estimated using a method based on continuous WT (CWT) modulus maxima detection (Figure 1)[11]. For each lead, CWT was applied on the unfiltered signals using a negative first-order Gaussian mother wavelet (i.e. first derivative of a Gaussian curve) along 40 linearly spaced

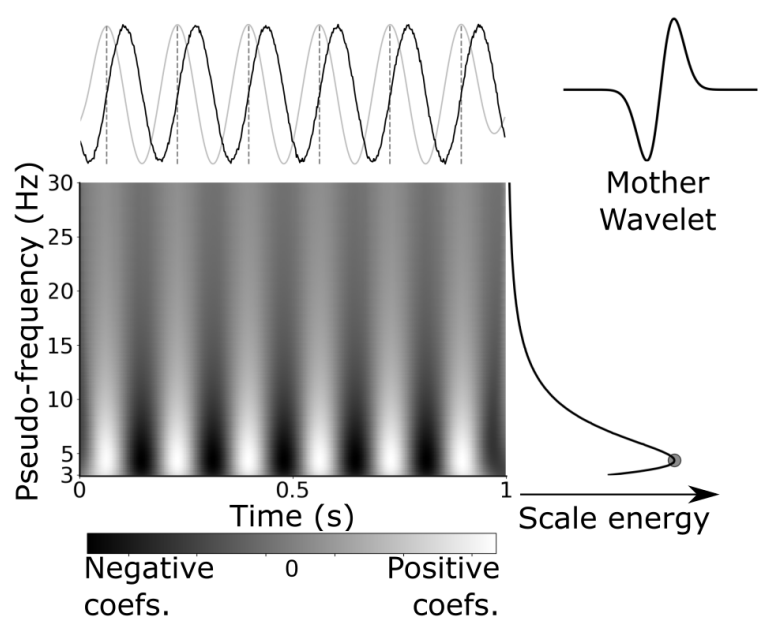

Figure 1. Wavelet method for DF detection. Peaks detected in the CWT performed with a negative Gaussian wavelet in the scale with highest energy are used to estimate cycle lengths and DFs.

scales in the pseudo-frequency range of 3 to $30 \mathrm{~Hz}$. In order to detect the most physiologically relevant positive deflections, the scale with the highest energy was selected for further processing; the rationale is that this scale contains the most information about a signal and thus represents better its main properties, such as the activation patterns in a BSPM signal. All local maxima are detected on the scale with the maximum energy after applying a $30 \mathrm{~Hz}$ lowpass filter (4th order, Butterworth) to avoid high-frequency components unrelated to the arrhythmia that may appear after CWT. The average AF cycle length $(C L)$ was determined from the intervals between the detected peaks and used for the DF calculation $(D F=1 / C L)$.

DFs were estimated by both the proposed method and Welch periodogram. The latter was computed using $2.7 \mathrm{~s}$ segments with zero-padding to achieve a frequency step of $0.1 \mathrm{~Hz}$ (i.e. $10 \cdot f_{S}$ samples), Hanning windows and overlap of $90 \%$. Torso DFs were determined by identifying the peak with the highest power in the periodograms withing the $\mathrm{AF}$ physiological frequency range $(2-15 \mathrm{~Hz})$ [12]. DF maps were generated for both methods by displaying the color coded DF values in the corresponding positions on torso. $\mathrm{HDF}_{\text {Atria }}$ values were estimated using the HDF from BSPM DF maps $\left(\mathrm{HDF}_{B S P M}\right)$. The methods' accuracy was assessed by calculating the absolute and relative errors between $\mathrm{HDF}_{B S P M}$ and $\mathrm{HDF}_{\text {Atria }}$; an estimation was considered correct if the absolute error was $\leq 1 \mathrm{~Hz}$.

The robustness of the $\mathrm{HDF}_{\text {Atria }}$ estimations for both methods was assessed using the simulated data with different levels of added WGN and harmonic noise, here simulated by adding a sinusoidal wave in the frequency of the first harmonic of $\mathrm{HDF}_{\text {Atria }}$ (SNR of 60,30, 10, 5 and 1 
$\mathrm{dB})$. To evaluate the robustness of both methods to lower spatial resolution BSPM lead layouts, the analyses were reproduced for subsets of the original lead layouts: 256 [13], 127[7], 67[6], 64[14], 32 and 16 for model data, and 32 and 16 leads for patients.

Results obtained with different methods were compared using Wilcoxon signed-rank test. For comparisons within the same method with different levels of noise or lead layouts, Friedman test was used (post-hoc: Nemenyi test).

\section{Results}

Fig 2A-D show a comparison of the Welch and wavelet methodologies in a patient with the driver region in the left atrium. The DF maps generated by both methods (Fig. 2A) show smoother transitions between DFs with the wavelet method, which contains a region of high DFs that do not appear in the Welch map. Looking at segments obtained from the HDF region in the atria (Fig. 2B) and in the BSPM (Fig. 2C), it is possible to observe that the activation times detected by the wavelet method correspond to the most prominent repetitive patterns, with cycle lengths similar to those observed in the EGMs. The HDF estimate based on the activations is closer to the $\mathrm{HDF}_{\text {Atria }}$, while a higher peak in the spectrogram, unrelated to the $\mathrm{HDF}_{\text {Atria }}$, increases the error in the Welch method (Fig. 2D).

The wavelet method outperformed the Welch approach in models and patients. Considering all AF simulations, the wavelet method achieved an relative error (median $[I Q R]$ ) of $7.14[3.06-16.23] \%$ vs. 60.00 [6.03$69.36] \%$ using Welch periodograms, $p=0.06$ (Fig. 2E, left). For patients, the errors were $8.10[5.50-13.14] \%$ vs. 7.47 [5.59-19.62]\% for both methods, respectively, $p=0.06$. Overall, the wavelet method resulted in correct estimations of $\mathrm{HDF}_{\text {Atria }}$ in $81.82 \%$ of the simulations, against $45.45 \%$ for Welch's estimates. In patients, the correct estimations occurred in $75.00 \%$ vs. $66.67 \%$ of the cases for both methods, respectively.

Fig. 3 shows the effects of the artifacts in the results from model (Fig. 3A-C) and patient data (D). The wavelet method is robust against Gaussian and harmonic noise, achieving lower mean and median errors than the Welch approach in every scenario. Significant differences in the estimations of the $\mathrm{HDF}_{\text {Atria }}$ appeared for the wavelet method between the $1 \mathrm{~dB}$ and the 30 and $60 \mathrm{~dB}$ SNRs for the harmonic noise $(p<0.01)$. For the Welch method, significant differences appeared only with harmonic noise between the $1 \mathrm{~dB}$ and the 60 and $30 \mathrm{~dB}$ SNRs $(p<0.05)$.

Both approaches allowed identifying the HDF even when reducing the amount of recorded leads to 16 in patients and models, achieving similar results along all lead layouts, with no significant differences $(p=0.23$ and $p=0.33$ for Welch and wavelet methods, respectively, with model data and $p=0.43$ and $p=0.79$ with patient

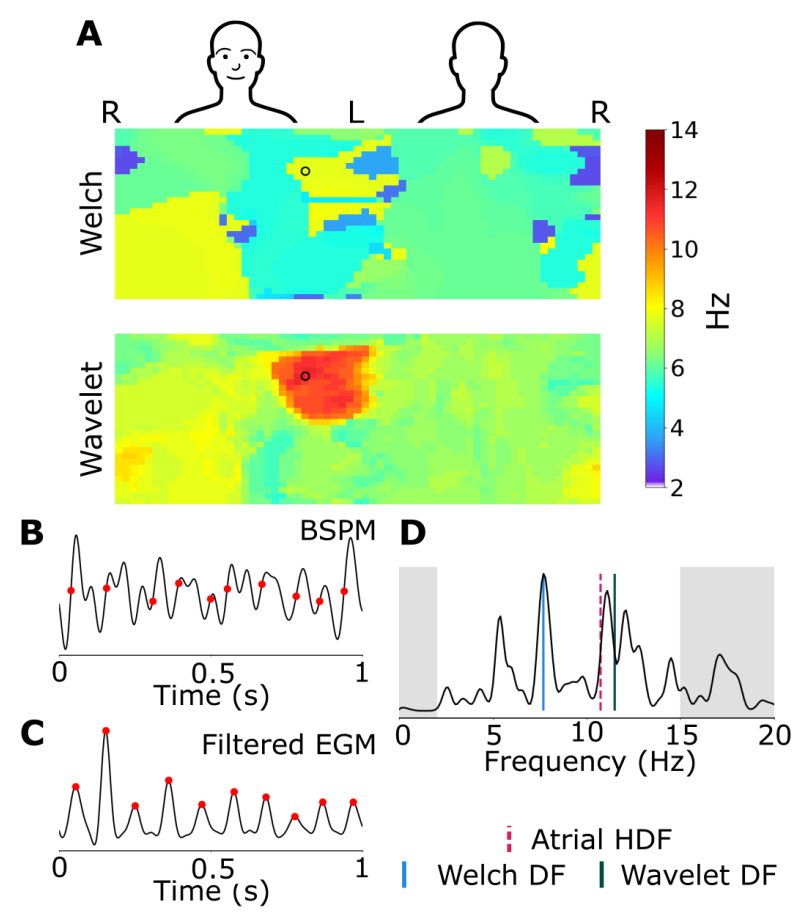

Figure 2. Comparison of the methods. A: DF maps from a patient with $\mathrm{HDF}_{\text {Atria }}$ in the left atrium; $\mathbf{B}$ and $\mathbf{C}$ : Excerpts of an EGM and a BSPM signal from the respective HDF regions; activations are marked in red; D: Welch periodogram of the signal in $\mathrm{B}$.

data). Errors obtained with the wavelet method are consistently lower than those from the Welch approach.

\section{Discussion and Conclusions}

In this study, a method based on maximum detection in CWT with Gaussian wavelets for the estimation of the DF in BSPM signals has been developed and compared with the traditionally widely applied approach based on peak detection in Welch periodograms. From the results it can be highlighted that the proposed wavelet-based method showed a better accuracy for estimating the arrhythmic mechanism frequency, even under noisy conditions and relative low number of layout leads. Preciser estimations can enhance the diagnostic ability of the DF analyses in BSPM, increasing the value of the BSPM as a low complexity tool for early diagnosis and follow-up.

DF analysis has been introduced in the study of AF as a means to provide a robust periodicity measurement of the fibrillatory waves [5]. However, frequency analysis is subject to uncertainties that may significantly alter the resulting DF [5]. WT is an intermediary technique between pure time or frequency analyses, providing good localization in both domains, which is incorporated in our method: the individual wavefronts are identified, while the selection of 

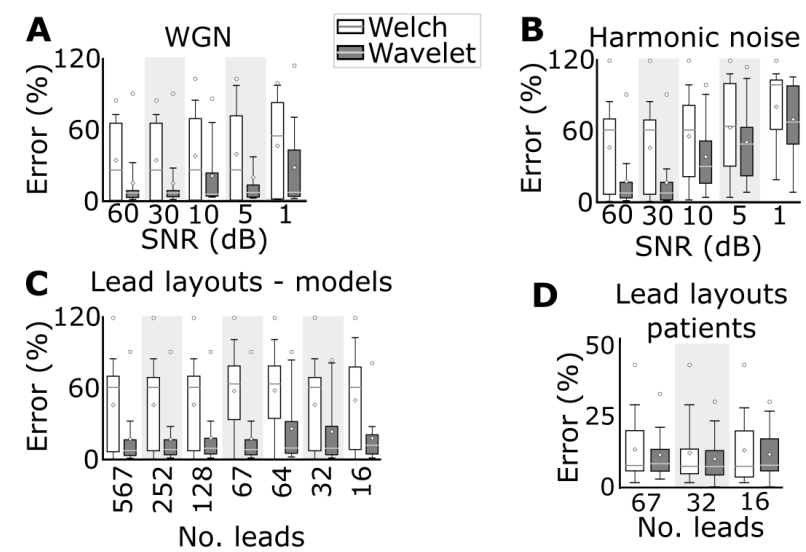

Figure 3. Atrial HDF error of the methods for different scenarios in models (A-C) and patients (D). Diamonds: means; lines: medians; whiskers: $5 \%$ and $95 \%$ percentiles

the scale with the highest energy in the AF physiological range acts as a sort of DF selection, focusing on the dominant periodic characteristics, but not restricting the analysis to a single frequency component. The method avoids derivative-based detection of activations [10], which has a high-pass characteristic that may lead to double counting due to the multiple inflections of the AF signal [11].

In addition to the increased accuracy in the estimation of $\mathrm{HDF}_{\text {Atria }}$, the proposed methodology has the potential to aid studies in the time and phase domains. The detection of wavefronts can be used for studying propagation patterns on the torso [10], and the estimation of instantaneous CLs can be used for detrending signals [11] or for estimating more precise frequencies for narrow band-pass filtering [9] prior to phase analysis based on Hilbert transform.

\section{Acknowledgments}

This study was supported in part by grants from São Paulo Research Foundation (2017/19775-3), Instituto de Salud Carlos III FEDER (Fondo Europeo de Desarrollo Regional PI17/01106) and Generalitat Valenciana Grants (AICO/2018/267).

\section{References}

[1] Kirchhof P, Benussi S, Kotecha D, Ahlsson A, Atar D, Casadei B, et al. 2016 esc guidelines for the management of atrial fibrillation developed in collaboration with eacts. Eur J Cardio Thorac 2016;50(5):e1-e88.

[2] Atienza F, Almendral J, Ormaetxe JM, Moya n, MartínezAlday JD, Hernández-Madrid A, et al. Comparison of radiofrequency catheter ablation of drivers and circumferential pulmonary vein isolation in atrial fibrillation: a noninferiority randomized multicenter radar-af trial. J Am Coll Cardiol 2014;64(23):2455-2467.
[3] Narayan SM, Krummen DE, Shivkumar K, Clopton P, Rappel WJ, Miller JM. Treatment of atrial fibrillation by the ablation of localized sources: CONFIRM trial. J Am Coll Cardiol 2012;60(7):628-636.

[4] Jalife J, Berenfeld O, Mansour M. Mother rotors and fibrillatory conduction: a mechanism of atrial fibrillation. Cardiovasc Res 2002;54(2):204-216.

[5] $\mathrm{Ng} \mathrm{J}$, Goldberger JJ. Understanding and interpreting dominant frequency analysis of af electrograms. J Cardiovasc Electrophysiol 2007;18(6):680-685.

[6] Guillem MS, Climent AM, Millet J, Arenal n, FernándezAvilés F, Jalife J, et al. Noninvasive localization of maximal frequency sites of atrial fibrillation by body surface potential mapping. Circ Arrhythm Electrophysiol 2013; 6(2):294-301.

[7] Vanheusden FJ, Chu GS, Li X, Salinet J, Almeida TP, Dastagir N, Stafford PJ, Ng GA, Schlindwein FS. Systematic differences of non-invasive dominant frequency estimation compared to invasive dominant frequency estimation in atrial fibrillation. Comput Biol Med 2019;104:299-309.

[8] Romero I, Fleck E, Kriatselis C. Frequency analysis of atrial fibrillation surface and intracardiac electrograms during pulmonary vein isolation. Europace 2011;13(9):1340 1345.

[9] Rodrigo M, Climent AM, Liberos A, Fernández-Avilés F, Berenfeld O, Atienza F, Guillem MS. Technical considerations on phase mapping for identification of atrial reentrant activity in direct-and inverse-computed electrograms. Circ Arrhythm Electrophysiol 2017;10(9):e005008.

[10] Guillem MS, Climent AM, Castells F, Husser D, Millet J, Arya A, Piorkowski C, Bollmann A. Noninvasive mapping of human atrial fibrillation. J Cardiovasc Electrophysiol 2009;20(5):507-513.

[11] Vijayakumar R, Vasireddi SK, Cuculich PS, Faddis MN, Rudy Y. Methodology considerations in phase mapping of human cardiac arrhythmias. Circ Arrhythm Electrophysiol 2016;9(11):e004409.

[12] Salinet JL, Tuan JH, Sandilands AJ, Stafford PJ, Schlindwein FS, Ng GA. Distinctive patterns of dominant frequency trajectory behavior in drug-refractory persistent atrial fibrillation: preliminary characterization of spatiotemporal instability. J Cardiovasc Electrophysiol 2014;25(4):371-379.

[13] Haïssaguerre M, Hocini M, Denis A, Shah AJ, Komatsu Y, Yamashita S, et al. Driver domains in persistent atrial fibrillation. Circulation 2014;130(7):530-538.

[14] Salinet JL, Marques VG, Mazzetto M, Camargo ED, Pastore CA, Cestari IA. A 64-lead body surface potential mapping system. In 2017 Computing in Cardiology (CinC). IEEE, 2017; 1-4.

Address for correspondence:

Victor Gonçalves Marques

Biomedical Engineering - CECS

Federal University of $\mathrm{ABC}$

E-mail: vgmarques.ufabc@gmail.com 